\title{
Risk factors for and management of sirolimus-associated pneumonitis in kidney transplant recipients
}

Original article Weiner SM et al. (2007) Pneumonitis associated with sirolimus: clinical characteristics, risk factors and outcome-a single-centre experience and review of the literature. Nephrol Dial Transplant 22: 3631-3637

\section{SYNOPSIS}

KEYWORDS dose, pneumonitis,

renal transplantation, risk factor, sirolimus

\section{BACKGROUND}

Cases of unexplained interstitial pneumonitis have become more frequent since the introduction of sirolimus for immunosuppression after renal transplantation.

\section{OBJECTIVES}

To pinpoint risk factors for pneumonitis in a series of kidney, kidney-pancreas or pancreas transplant patients receiving sirolimus, and to report the outcomes of treatment.

\section{DESIGN AND INTERVENTION}

The medical records of patients who received sirolimus after kidney, kidney-pancreas or pancreas transplantation at a German center were reviewed to identify cases of pneumonitis. A review of published papers reporting more than one case of potentially sirolimus-related pulmonary toxicity was also carried out.

\section{OUTCOME MEASURE}

The end point was suspected sirolimusassociated pneumonitis.

\section{RESULTS}

Of the 115 patients who received sirolimus (81 kidney transplant recipients, 33 kidneypancreas transplant recipients and 1 pancreas transplant recipient), 11 (9.6\%) developed interstitial pneumonitis. The affected patients had initiated sirolimus 1-8 months before the development of pneumonitis. Sirolimus was combined with low-dose (2-10 mg/day) prednisone in all cases; one patient additionally received tacrolimus and one patient also received ciclosporin. None of the affected patients had received sirolimus as de novo immunosuppression. Presenting symptoms included low-grade fever $(n=8)$, dry cough $(n=7)$, exertional dyspnea $(n=4)$, and fatigue $(n=3)$. The patients who developed pneumonitis included the same proportion of males as those who did not develop pneumonitis (64\%), and were of a similar mean age (52.6 years vs 47.5 years). The mean trough sirolimus blood level, as measured by immunoassay, was $16.7 \mu \mathrm{g} / \mathrm{l}$ (range $6.2-38.7 \mu \mathrm{g} / \mathrm{l}$ ) at diagnosis of pneumonitis. Patients who developed pneumonitis had a significantly lower mean glomerular filtration rate (GFR; as estimated by the Modification of Diet in Renal Disease formula) than those who did not develop pneumonitis $(21.3 \mathrm{ml} / \mathrm{min}$ vs $38.7 \mathrm{ml} / \mathrm{min}$; $P=0.002)$. Hemodialysis was required by two patients shortly before diagnosis of pneumonitis. In the multivariate analysis, only serum creatinine level and GFR were independent risk factors for pneumonitis $(P=0.014$ and $P=0.020$, respectively). Patients with pneumonitis underwent discontinuation of sirolimus $(n=5)$ or reduction of their sirolimus dose $(n=6)$; antibiotics were given to 10 of these patients but had no clear benefit. All cases of pneumonitis resolved within 2-4 weeks. During a mean follow-up of 29 months (range 15-48 months), one patient whose sirolimus dose was reduced had a relapse of pneumonitis (after 5 months), necessitating discontinuation of the drug. Combined analysis of these 11 cases and 50 cases previously reported in the literature revealed that late switch to sirolimus was associated with a higher frequency of pneumonitis than was de novo sirolimus use $(14.1 \%$ vs $3.8 \% ; P=0.0024)$.

\section{CONCLUSION}

Late switch to sirolimus and compromised renal function might be associated with an increased likelihood of sirolimus-associated pneumonitis after kidney transplantation; there is a risk of relapse if sirolimus is continued. 


\section{COMMENTARY}

\section{Stuart M Flechner}

Weiner et al. review recent clinical information on the development of pulmonary toxicity in renal, renal-pancreas or pancreas transplant recipients given sirolimus. The authors point out that the 11 cases of pneumonitis they recorded seemed to cluster among recipients switched to sirolimus from a calcineurin inhibitor, those with raised sirolimus $\mathrm{C}_{0}$ levels $(>15 \mathrm{ng} / \mathrm{ml})$, and those with substantial renal impairment (estimated GFR $<22 \mathrm{ml} / \mathrm{min}$ ). No pulmonary toxicity was noted among 35 patients given sirolimus de novo. Importantly, symptoms of pneumonitis resolved 14-28 days after discontinuation of sirolimus (carried out in 5 patients) or reduction of sirolimus dose (carried out in 6 patients). This observation implies a role for increased blood levels of sirolimus in many instances of pulmonary toxicity. Even in the 5 patients who discontinued sirolimus, it would be extremely unlikely that the drug was completely cleared by the time symptoms resolved (elimination half life 2-3 days). ${ }^{1}$

The authors tabulated a total of 61 cases of probable sirolimus-induced pulmonary toxicity from the literature, including their 11 cases. The role of sirolimus in the additional 50 cases of noninfectious pneumonitis is complicated by the use of other agents such as antibodies, azathioprine, and mycophenolate mofetil, which have also been implicated in inflammation of lung tissue. ${ }^{2-4}$ All but a few of the case patients presented with raised sirolimus blood levels (mean $16.2 \mathrm{ng} / \mathrm{ml}$ ), and a greater proportion of cases occurred after conversion to sirolimus from a calcineurin inhibitor than from de novo use ( $14 \%$ vs $4 \%$; $P=0.0024)$. The episodes of pneumonitis occurred as soon as 3 days and as late as 4 years after the introduction of sirolimus. Interestingly, pulmonary toxicity was not reported in a study of 50 kidney transplant patients or in a study of 27 liver transplant patients, both of which trials included 2-3 years of sirolimus monotherapy. ${ }^{5,6}$

Whether sirolimus causes primary injury to lung tissue or exacerbates pre-existing inflammation or infection (or both) is not known. Some authors have suggested that sirolimus causes drug-induced hypersensitivity. Mammalian target of rapamycin (mTOR) inhibitors such as sirolimus are known to exacerbate edema in inflamed tissue by degrading the healing process and promoting capillary leakage. Influenza A virus and Mycoplasma were detected via molecular techniques in 2 of the 11 cases reported by Weiner and colleagues, but were dismissed as factors that contribute to pneumonitis. Pulmonary toxicity might not be common to all mTOR agents, but is possibly drug-specific. ${ }^{7}$

Pulmonary symptoms in any organ transplant recipient demand prompt attention to identify any causative pathogens. In addition, clinical judgment is needed to distinguish individuals with mild symptoms from those who are hypoxic, have considerable consolidation, or have acute respiratory distress syndrome. For patients on sirolimus, especially those who have been switched from a calcineurin inhibitor because of renal dysfunction or those who are also receiving azathioprine or mycophenolate, it would seem prudent to either discontinue sirolimus or lower $\mathrm{C}_{0}$ levels to $5-8 \mathrm{ng} / \mathrm{ml}$ if pulmonary symptoms are mild. If the clinical picture is more severe, complete discontinuation is warranted until symptoms resolve.

\section{References}

1 Stenton SB et al. (2005) Sirolimus: the evidence for clinical pharmacokinetic monitoring. Clin Pharmacokinet 44: 769-786

2 Shrestha NK et al. (2003) Pneumonitis associated with the use of mycophenolate mofetil. Transplantation 75: 1762

3 Ananthakrishnan AN et al. (2007) Severe pulmonary toxicity after azathioprine/6-mercaptopurine initiation for the treatment of inflammatory bowel disease. J Clin Gastroenterol 41: 682-688

4 Bamgbola FO et al. (2003) Non-cardiogenic pulmonary edema during basiliximab induction in three adolescent renal transplant patients. Pediatr Transplant 7: 315-320

5 Arellano EM et al. (2007) Sirolimus monotherapy as maintenance immunosuppression: single-center experience in 50 kidney transplant patients. Transplant Proc 39: 2131-2134

6 Di Benedetto F et al. (2007) Sirolimus monotherapy in liver transplantation. Transplant Proc 39: 1930-1932

7 De Simone P et al. (2007) Switch to everolimus for sirolimus-induced pneumonitis in a liver transplant recipient-not all proliferation signal inhibitors are the same: a case report. Transplant Proc 39: 3500-3501

SM Flechner is a Professor of Surgery at Cleveland Clinic Lerner College of Medicine, Cleveland, $\mathrm{OH}$, USA.
Acknowledgments

The synopsis was written by Chloë Harman, Locum Editor,

Nature Clinical Practice.

\section{Competing interests}

The author has declared associations with the following companies: Bristol-Myers Squibb Novartis, Roche and Wyeth. See the article online for full details of the relationships.

\section{Correspondence}

Cleveland Clinic 9500 Euclid Avenue, A110

Cleveland

$\mathrm{OH} 44195$

USA

flechns@ccf.org

Received 31 December 2007 Accepted 17 January 2008 Published online 26 February 2008

www.nature.com/clinicalpractice doi:10.1038/ncpneph0768

PRACTICE POINT Pulmonary symptoms in a renal transplant recipient on sirolimus should prompt discontinuation of this agent or reduction of $\mathrm{C}_{0}$ levels to $5-8 \mathrm{ng} / \mathrm{ml}$, depending on the severity of symptoms 